\title{
Simulation of Npn and Pnp AlGaN/GaN heterojunction bipolar transistors performances: limiting factors and optimum design
}

C. Monier*, F. Ren, J. Han, P. C. Chang, R. J. Shul, K. P. Lee, A. P. Zhang, A. G. Baca, and S. J. Pearton.

- C. Monier, K. P. Lee, and S. J. Pearton are with the Department of Materials Science and Engineering, University of Florida, Gainesville, Florida 32611

- F. Ren, and A. P. Zhang are with the Department of Chemical Engineering, University of Florida, Gainesville, Florida 32611.

- J. Han, P. C. Chang, R. J. Shul, and A. G. Baca are with Sandia National Laboratories, Albuquerque, New Mexico 87185.

*: Current address at Sandia National Laboratories, Electronic mail: cmonier@sandia.gov

\section{Abstract}

The performance capabilities of $N p n$ and Pnp AlGaN/GaN heterojunction bipolar transistors

have been investigated by using a drift-diffusion transport model. Numerical results have been employed to study the effect of the $p$-type $\mathrm{Mg}$ doping and its incomplete ionization on device performance. The high base resistance induced by the deep acceptor level is found to be the cause of limited current gain values for $\mathrm{Npn}$ devices. Several computation approaches have been considered to improve their performance. Reasonable improvement of the DC current gain $\beta$ is observed by realistically reducing the base thickness in accordance with processing limitations. Base transport enhancement is also predicted by the introduction of a quasi-electric field in the base. The impact of the base resistivity on high-frequency characteristics is investigated for $N p n$ AlGaN/GaN devices. Optimized predictions with maximum oscillation frequency value as high as $f_{\text {MAX }}=20 \mathrm{GHz}$ and a unilateral power gain $-U=25 \mathrm{~dB}$ make this bipolar GaN-based technology compatible with communication applications. Simulation results reveal that the restricted amount of free carriers from 


\section{DISCLAIMER}

This report was prepared as an account of work sponsored by an agency of the United States Government. Neither the United States Government nor any agency thereof, nor any of their employees, make any warranty, express or implied, or assumes any legal liability or responsibility for the accuracy, completeness, or usefulness of any information, apparatus, product, or process disclosed, or represents that its use would not infringe privately owned rights. Reference herein to any specific commercial product, process, or service by trade name, trademark, manufacturer, or otherwise does not necessarily constitute or imply its endorsement, recommendation, or favoring by the United States Government or any agency thereof. The views and opinions of authors expressed herein do not necessarily state or reflect those of the United States Government or any agency thereof. 


\section{DISCLAIMER}

Portions of this document may be illegible in electronic image products. Images are produced from the best available original document. 
the $p$-doped emitter limits Pnp's DC performances operating in common emitter configuration. A preliminary analysis of r.f. characteristics for the $P n p$ counterpart indicates limited performance mainly caused by the degraded hole mobility.

\section{Index terms - $\quad \mathrm{AlGaN} / \mathrm{GaN} \mathrm{HBT}$}

Simulation

$\mathrm{Mg}$ ionization efficiency

Base resistivity

Quasi-electric field

DC current gain

High frequency characteristics 


\section{Introduction}

GaN-based materials have recently gained considerable interest for their use in optoelectronic devices with a variety of spectral ranges. The unique combination of wide bandgap IInitride material characteristics, such as large breakdown field or high electron saturation velocity, makes $\mathrm{GaN}$ a suitable candidate for electronic devices intended for high power, high frequency applications. Impressive reports [1] in the past few years on AlGaN/GaN High Mobility Electron Transistors (HEMTs) have motivated the development of heterojunction bipolar transistors (HBTs) which have demonstrated, in GaAs and InP, improved linearity and more uniform threshold voltages over the field effect transistor counterpart. The first $N p n$ AlGaN/GaN HBTs have been recently reported $[2,3]$. Initial current gain $\beta$ values were measured to be as high as 3 at room temperature. Poor conductivity of the p-type GaN base layer, due to the high acceptor ionization energy (>170 $\mathrm{meV}$ ) is responsible for the current gain limitation [3]. Others factors including $\mathrm{Mg}$ memory effect (associated with substantial hole diffusion to the emitter) or the conflicting effect of the large spontaneous polarization (inherited from typical MOCVD-grown Ga-face heterostructures along the [0001] direction) have also been considered to explain the moderate gain of the devices [4,5]. Both aspects will result in reduced free hole concentration and artificially larger thickness for the base layer. Solutions involving the use of $\mathrm{AlGaN} / \mathrm{GaN}$ superlattices [6] or piezoelectric effects associated with modulation-doped heterostructures and emitter-up HBT configuration [7] have been suggested to achieve higher free hole concentration in the base. More recently, AlGaN/GaN Pnp HBTs have been fabricated as well [8] with devices operated in the common base bias configuration.

Because GaN-based electronics are still in their infancy, there has been very little work on simulating HBTs capabilities in these materials $[9,10]$. In this paper, we report on the simulation results of $N p n$ and Pnp.GaN-based HBTs for use in optimizing the epitaxial multilayer structure and in assessing the factors limiting/maximizing device performance for power applications. After a description of the two-dimensional formalism used in this work. the initial performances of the Npn 
HBT structure recently demonstrated are simulated to verify the validity of the numerical approach and the material parameter choice. Unlike previous works that did not pay too much attention to the $\mathrm{Mg}$ ionization effect, simulation results are explored here to evaluate the free hole concentration issue for both $N p n$ and $P_{n p}$ configurations. Solutions to overcome these limitations are presented, leading to enhanced DC performance. Finally, simulated high-frequency characteristics are presented for the two types of HBTs in the light of these optimized designs.

\section{Numerical approach and GaN parameter consideration}

We have simulated the operation of the GaN-based HBT using a program based on the driftdiffusion model [11]. Performance analysis was achieved by self-consistent solution of the Poisson, carrier continuity and current density equations of a two-dimensional structure. Physical models incorporated in the simulation include carrier statistics, low-field mobility and generationrecombination mechanisms. A typical conduction band offset ratio $Q_{c}$ of 0.70 was employed for the energy band gap discontinuity at the AlGaN-GaN emitter/base interface [5]. The specific contact resistances for both $n$ and $p$-type materials are also specified according to recent reports with improved characteristics $[12,13]$. A value of $1 \times 10^{-6} \Omega / \mathrm{cm}^{-2}$ was used for the $n$-type ohmic contact while the $p$-type distributed resistance was assumed to be $5 \times 10^{-4} \Omega / \mathrm{cm}^{-2}$. Because the thickness of the AlGaN emitter layer is as large as $1500 \AA$, the effect of the piezoelectric polarization has not been introduced at the interface between the $\mathrm{GaN}$ base and the fully relaxed $\mathrm{AlGaN}$ top layer.

While modeling of HBTs has been ongoing in conventional III-V InP/InGaAs or AlGaAs/GaAs systems with well-known material characteristics and parameters, some fundamental characteristics of III-nitride based materials remain uncertain or strongly material quality-dependent. Simulations have been performed using experimental minority carrier mobility and lifetime values resulting from a carefuit analysis of data available in the literature $[14,15]$. Concentration dependent low-field mobilities range from 125 to $800 \mathrm{~cm}^{2} / \mathrm{V}$.s for the different electron concentrations typically 
considered through the structure. Much lower values were used for $p$-type nitride materials, ranging between 5 and $20 \mathrm{~cm}^{2} /$ V.s. Minority carrier lifetimes of $6.5 \mathrm{~ns}$ and $0.1 \mathrm{~ns}$ have been respectively reported from electron beam induced current measurements for $n$-type and $p$-type GaN materials with carrier concentration in the $10^{17} \mathrm{~cm}^{-3}$ range [15]. Since no systematic measurement of lifetime as a function of doping levels was reported, an interpolation method has been used to specify their value through the entire device structure. We assumed mobility and lifetime values in AlGaN materials to be identical to the ones extracted from binary GaN due to the lack of experimental measurements related to the III-nitride ternary alloy. Calculations also account for incomplete impurity ionization in the p-type $\mathrm{GaN}$ base layer. The ionized acceptor $(\mathrm{Mg})$ impurity concentration is given by the classical Fermi-Dirac statistics with appropriate acceptor energy level $\mathrm{E}_{\mathrm{A}}$ and operating temperature. The activated carrier concentration in $n$-type materials is assumed equal to the doping concentration since Si-doped layers do not suffer from a large donor activation energy ( $\left.E_{D} \sim 15 \mathrm{meV}\right)$.

\section{Mg ionization efficiency issue on DC performances}

\subsection{Npn AlGaN/GaN HBT}

The initial Npn AlGaN/GaN structure consists of a $0.5 \mu \mathrm{m}$, Si-doped $\left(10^{17} \mathrm{~cm}^{-3}\right) \mathrm{GaN}$ collector followed by a $0.15 \mu \mathrm{m}, M g$-doped (with free hole concentration<1 $\times 10^{18} \mathrm{~cm}^{-3}$ for a doping concentration in the $10^{20} \mathrm{~cm}^{-3}$ range) $\mathrm{GaN}$ base and a $0.1 \mu \mathrm{m}, S i$-doped $\left(5 \times 10^{17} \mathrm{~cm}^{-3}\right) \mathrm{Al}_{0.15} \mathrm{Ga}_{0.85} \mathrm{~N}$ emitter. The band gap of the ternary material is fixed to $E_{G}=3.85 \mathrm{eV}$. The geometrical design of this vertical mesa architecture exhibits an active emitter length of $3 \mu \mathrm{m}$ and a base length of $6 \mu \mathrm{m}$. Fig. 1(a) shows calculated Gummel plots in the common emitter configuration for $N p n$ 's operating at $25^{\circ} \mathrm{C}$. Several acceptor activation energies are considered through an arbitrary parameter $E_{A}$ to describe distinct incomplete ionization conditions. The $\mathrm{E}_{\mathrm{A}}$ parameter variation is used as a means of considering the inclination for $\mathrm{Mg}$ species to exhibit low activation in the base. 
Because of the uncertainty related to minority carrier lifetime and mobility values and their strong dependence on the GaN material quality, a relative comparison between numerical results is considered to understand the various elements affecting the device performances. No significant differences are observed with base-emitter voltage value $V_{B E}$ up to $3.5 \mathrm{~V}$, except that the base current $I_{B}$ is more sensitive to the free hole concentration and is always larger than the collector output $I_{C}$. As $V_{B E}$ increases, $I_{C}$ is strongly affected by the free hole carrier concentration. Devices with poor $\mathrm{Mg}$ ionization efficiency (as low as $0.01 \%$ activated carriers) exhibit an $\mathrm{I}_{C}$ output lower by about one order of magnitude compared to devices with the highest activated doping species (as high as $20 \%$ ). The DC gain is shown in Fig. 1(b) as a function of $\mathrm{V}_{\mathrm{BE}}$ for the same previous set of activation energies. Working HBTs with $\beta>1$ can be realized with moderate $V_{B E}$ voltage when the free hole concentration is not too degraded. An increasing "threshold" voltage for gain higher than 1 is observed as the $\mathrm{Mg}$ activation is reduced. No amplification gain can ultimately be achieved unless an extremely high but unrealistic $V_{B E}$ bias is applied. These results are analyzed in terms of improved base resistance. A highly resistive base layer induced by the deep $\mathrm{Mg}$ nature forces base current flowing directly to the collector, bypassing the base. These carriers are usually required to control the amount of the base-emitter bias, and the amount of electron injection from the emitter to the base. Hence, reducing the free hole carrier concentration in the base has a direct impact on the current collected at the collector. Improved experimental DC characteristics have been obtained in $\mathrm{Npn}$ GaNbased HBTs at elevated temperature $\left(300{ }^{\circ} \mathrm{C}\right)$ with $\beta$ values up to $10[3,4]$. Another practical way of producing an increased amount of holes is to consider the use of $\mathrm{GaN}$ spacer layers to limit the $\mathrm{Mg}$ diffusion. The contamination of the emitter by $\mathrm{Mg}$ acceptors also degrades the free hole density in the base, just as a reduced ionization efficiency does.

\subsection{Pnp AlGāN/GaN HBT}


There are several advantages to the Pnp configuration for AlGaN/GaN HBTs. The band discontinuity at the emitter-base interface is more advantageous to prevent back-injection of carriers. Secondly, the base resistance will be much lower due to higher doping level achievable in n-type material. Although GaN-based Pnp HBT's have not been demonstrated in common emitter configuration [8], these bias conditions are nonetheless considered here to provide a quantitative comparison with $N p n$ results. The Pnp structure employs a geometrical design similar to the $N p n$ counterpart. The emitter and the collector layers are assumed to be doped with $\mathrm{Mg}$ species and acceptor ionization energies are considered to adjust their hole concentration. An initial Mg doping concentration of $1 \times 10^{19} \mathrm{~cm}^{-3}$ is chosen for both collector and emitter layers with an identical acceptor binding energy. A $1500 \AA$ GaN base layer with a donor concentration of $2 \times 10^{18} \mathrm{~cm}^{-3}$ completes the structure. Low contact resistance values characterize the emitter and the collector while the base is no longer penalized by a contact resistivity value that is too large. Fig. 2 shows the Gummel plot of Pnp AlGaN/GaN HBTs and its associated DC current gain with different acceptor energies from 0.1 to $0.3 \mathrm{eV}$ to address the ionization efficiency issue. A comparable current behavior for $I_{C}$ and $I_{B}$ is observed at low current density with bias up to $V_{B E}=-3.5 \mathrm{~V}$. The moderate $\beta$ value of 5 is not degraded by hole lifetime values (one order of magnitude higher than electron lifetime used for $N p n$ 's $)$ but is strongly dependent on the hole mobility value in the base $\left(<10 \mathrm{~cm}^{2} / \mathrm{V} . \mathrm{s}\right)$. As the applied base bias increases, a saturation regime is established. This regime that dramatically affects the gain strongly depends on the activation energy value. The higher the $E_{A}$, the lower base-emitter bias at which this regime comes out due to the low free hole carrier density. Devices with high ionization efficiency can produce a decent gain value for high current density while a too low efficiency limits the use of Pnp's to a current density range unsuitable for high power applications. Increasing the free hole density in the emitter with a much higher doping concentration will not help overcome the existing ionization efficiency issue but will result on the contrary in completely depleting the base region. 


\section{Design solution for enhanced $N p n$ DC performances}

Different approaches have been considered to overcome the dual negative influence of high base resistance and reduced base lifetime/mobility on the current gain $\beta$. The influence of the base thickness $X_{B}$ must be included into this simulation work. Recombination mechanisms are dominant in $\mathrm{GaN}$ materials, and the current gain is expressed as the ratio of lifetime $\tau_{\mathrm{n}}$ to the minority carrier transit time $\tau_{\mathrm{b}}$ across the base (proportional to the square of $\mathrm{X}_{\mathrm{B}}$ ). In addition, any $\mathrm{Mg}$ diffusion mechanism into the emitter artificially increases the base thickness. Although a lower acceptor binding energy $E_{A}$ has not been demonstrated yet by the existing growth techniques, an enhanced free hole carrier concentration (with higher ionization efficiency) can be employed to approximate the lower tendency for $\mathrm{Mg}$ atoms diffusing into the $n$-type emitter. Note that modeling rigorously the effect of $\mathrm{Mg}$ diffusion might be more complicated. In first approximation, the combining effect of a reduced base thickness with a higher ionization efficiency (lower $E_{A}$ value) must reveal the ability of optimizing the carrier transport across the base and reproduce the limitation of $\mathrm{Mg}$ diffusion to the emitter layer.

Fig. 3 displays both collector and base output currents $I_{C}$ and $I_{B}$ as a function of the baseemitter voltage in the on-state region (for $\mathrm{V}_{\mathrm{BE}}>3.0 \mathrm{~V}$ ) for three different values of the base thickness $X_{B}(750,1000$ and the initial $1500 \AA)$. In addition to the thickness variation, one new set of parameters $\left(E_{\mathrm{A}}=120 \mathrm{meV}\right.$ and $\left.\rho=5 \times 10^{-5} \Omega / \mathrm{cm}^{-2}\right)$ is employed to simulate the case of a higher free hole concentration/limited $\mathrm{Mg}$ diffusion. An improved ionization efficiency not only contributes to an enhanced free hole concentration but also leads to a less resistive base distributed contact. From a strict transport point of view, substantial enhancement of the current gain by a factor of 3 is obtained by reducing the base from 1500 to $750 \AA$ as shown in Fig.3. Because more electrons are collected instead of recombining in the base, less holes are supplied from the base contact to fulfill these recombination events and the base current output is continuously reduced in the present $X_{B}$ range. In 
the same way, the current gain enhancement by a factor of 4 to 5 observed with reduced base thickness and higher free hole carrier concentration symbolizes the expected DC performance HBTs could demonstrate without $\mathrm{Mg}$ contamination of the emitter. Note that from the $\beta$ consideration alone, the design decision is to make the base as thin as possible, but thick enough so that etching down and the subsequent metal deposition onto the base layer are still made reliably. However, the existing high base resistivity in the p-type $\mathrm{GaN}$ base layer, induced by the poor $\mathrm{Mg}$ ionization efficiency, has to be considered for further rf performances. If the base layer is made excessively thin, the increased base resistance may result in unacceptably low r.f characteristics.

One way to keep the base thick enough is to enhance the base transport by the introduction of a quasi-electric field in the base. This field is practically established by grading the aluminum concentration in the base layer (with the higher $\mathrm{Al}$ content placed near the emitter side). Simulations have been performed for various graded conduction band profiles associated with electric field values from $\mathrm{E}_{\mathrm{F}}=0$ (no gradual $\mathrm{Al}$ composition) up to $30 \mathrm{kV} / \mathrm{cm}(\sim 12 \%$ gradual $\mathrm{Al}$ composition) and results are shown in Fig. 4 where $I_{C}$ and $I_{B}$ are displayed over the on-state region. The base thickness is chosen to be $X_{B}=1500 \AA$ and the ionization efficiency is kept as it was initially $\left(E_{A}=170\right.$ meV). Note that collector and base current outputs decrease with increasing quasi-electric field values for low bias $\left(\mathrm{V}_{\mathrm{BE}}<3.5 \mathrm{~V}\right)$. This can be explained by the extra potential step in the conduction band (in addition to the spike peak characterizing abrupt HBTs) induced by the graded band structure that carriers have to overcome at the emitter-base interface to be injected in the base. Consequently the turn-on voltage is slightly larger as the grading $\mathrm{Al}$ composition increases in the base. As $\mathrm{V}_{\mathrm{BE}}$ increases, significant improvement of the $I_{C}$ value is predicted compared to the non-graded structure. The application of a higher forward bias reduces the potential barrier so that more carriers can be injected in the base and then accelerated by the presence of the field producing higher $\mathrm{I}_{\mathrm{C}}$ values. By combining the reduced base layer/enhanced free hole concentration effect with a large quasi-electric field, an improvement of the current gain by a factor of 10 is ultimately observed. 


\section{Predicted high-frequency performances}

In addition to DC current gain, the small signal gain $h_{2 l}$ and the unilateral power gain $U$ have been calculated as a function of the frequency for several operating conditions. Simulated r.f. performances are obtained by calculating conductance/capacitance between each pair of electrodes and the induced $S$-parameters at any given DC bias point. The corresponding cutoff frequency, $f_{\tau}$, and maximum oscillation frequency, $f_{\mathrm{MAX}}$, have been extrapolated as figures of merit for highfrequency characteristics. Predictions have previously evaluated the AlGaN/GaN high-frequency capability with $f_{\mathrm{T}}$ and $f_{\operatorname{MAX}}$ values as high as 44 and $24 \mathrm{GHz}$ [9]. The simulation approach is used in this present work to investigate the impact of the base resistivity on high-frequency characteristics. Fig. 5 displays simulated small-signal performances for a $N p n$ AlGaN/GaN $\mathrm{HBT}$, with a conventional base thickness of $1500 \AA$, a $\mathrm{Mg}$ ionization energy of $170 \mathrm{meV}$ and $\mathrm{DC}$ bias conditions of $\mathrm{V}_{\mathrm{BE}}=6 \mathrm{~V}$ and $\mathrm{V}_{\mathrm{CE}}=16 \mathrm{~V}$. Once again, the uncertain minority carrier lifetime and mobility values impose a relative analysis of the high-frequency characteristics to be adopted. The nonexistence of experimental r.f. values implies a certain vigilance for the interpretation of the present simulated results. The $f_{\text {MAX }}$ value of $10 \mathrm{GHz}$ is found to be lower than $f_{T}$ (not displayed in the chart but equal to $25 \mathrm{GHz}$ ). The high base resistance is thought to be the cause of this result. The problematic ionization efficiency of $\mathrm{Mg}$ acceptors not only gives rise to high resistivity of $p$-type GaN layers but also makes it difficult to realize $p$-ohmic contacts with low resistance. Predictions for devices with different base contact resistance are shown in Fig.5. The collector current density is fixed at the same value. The collector-emitter bias remains unchanged. Hence, only the base transit time among all the various charging/transit times is affected and the isolated effect of the base resistance can be addressed. A significant variation of the $f_{\text {MAX }}$ value (from 4 to $15 \mathrm{GHz}$ ) is observed over the distributed contact resistance range demonstrating the critical influence of a high contact resistivity. The unilateral power gain value increases as well with lower contact resistance for more efficient high power characteristics. The $f_{\operatorname{MAX}}$ value degrades similarly by considering a variation of the base 
resistivity through lower ionization efficiency. Values as low as $3 \mathrm{GHz}$ are found, for instance, with devices that differ from the initial one (with $f_{\mathrm{MAX}}=10 \mathrm{GHz}$ ) by an $\mathrm{E}_{\mathrm{A}}$ value of $300 \mathrm{meV}$. Improved $f_{\text {MAX }}$ values are demonstrated by increasing the $\mathrm{V}_{\text {CE }}$ value to $40 \mathrm{~V}$ as shown in Fig. 5. This high operation voltage value at the base-collector junction is easily achievable due to the typically large GaN breakdown critical field and the low collector doping of this particular design. Npn HBT structures including improved $\mathrm{Mg}$ ionization efficiency, optimized bias conditions and the use of a compositionally graded base layer eventually lead to $f_{\mathrm{MAX}}$ around $20 \mathrm{GHz}$ and a unilateral power gain $U=25 \mathrm{~dB}$. The above results demonstrate the feasibility of using GaN-based Npn's for radar and communication applications working in the $5 \mathrm{GHz}$ frequency range.

Less impressive values are demonstrated when small-signal analysis is undertaken on $P n p$ AlGaN/GaN HBTs. Predicted maximum oscillation frequencies reach a value of $f_{\mathrm{T}}=2 \mathrm{GHz}$ as shown in Fig. 5, which is one order of magnitude less than a similarly designed $\mathrm{Npn}$ AlGaN/GaN device. The actual limitation is mainly related to the general difficulty to achieve high hole mobility values for $\mathrm{GaN}$ materials in the entire structure.

\section{Conclusion}

In summary, the performances of $N p n$ and Pnp AlGaN/GaN HBT's have been investigated with the use of a two-dimensional physically based device simulator to assess capabilities of these recently demonstrated devices. Simulation using experimental mobility and lifetime reported in the literature closely reproduce the Gummel plot from the Npn HBT structures recently demonstrated. The influence of the Mg ionization efficiency on the Npn GaN-based HBT has been theoretically addressed. The highly resistive base layer induced by the deep $\mathrm{Mg}$ energy level forces base current to flow directly to the collector. To overcome the critical influence of reduced lifetime and high base resistivity on the current gain $\beta$ in the highly dislocated base region, we have examined the effect of the base thickness and a compositional base grading (by introducing a quasi-field) to aid carrier 
transport across the base. Significant improvements in $\beta$ are predicted by combining a reasonably thin base layer with a large quasi-electric field. Simulated DC performances of the Pnp transistor in common emitter configuration reveal the intrinsic limitation for this device to achieve reasonable current gain value associated at high current density. This is because of the difficulty of providing enough free holes without doping the emitter excessively. An analysis of high frequency characteristics for Npn AlGaN/GaN HBTs indicates a substantial impact of the high base resistivity on predicted r.f. performances, which primarily affects the maximum oscillation frequency. Optimized transistor design and bias show a maximum oscillation frequency as high as $20 \mathrm{GHz}$ and a practical unilateral power gain of $25 \mathrm{~d} B$ that makes this device promising for microwave power amplifiers. These encouraging results are expected to improve as issues related to defects and impurities are better understood and resolved. Preliminary simulated performances on Pnp AlGaN/GaN HBTs always display frequency values one order of magnitude lower than the $N p n$ ones. A limited hole mobility is found to be responsible for this degradation.

\section{Acknowledgments}

The work at UF is partially supported by ONR grant N00014-98-1-0204 (J.C. Zolper) and a DARPA/EPRI Grant MDA-972-98-1-0006 (D. Radack/J. Melcher). Sandia is a multi-program laboratory operated by Sandia Corporation, a Lockheed-Martin company, for the US Department of Energy under Contract $N^{\circ}$ DE-AC04-94-AL-85000. 


\section{References}

[1] M. J. Murphy, K. Chu, H. Wu, W. Yeo, W. J. Schaff, O. Ambacher, L. F. Eastman, T. J. Eutsis, J. Silcox, R. Dimitrov, and M. Stutzmann, “ High-frequency polarization-induced HEMTs grown by plasma-assisted molecular beam epitaxy," Appl. Phys. Lett. 75, 3653 (1999)

[2] L. S. McCarthy, P. Kozodoy, M. J. W. Rodwell, S. P. DenBaars, and U. K. Mishra, "AlGaN/GaN heterojunction transistor," IEEE Electron Device Lett. 20, 277 (1999)

[3] F. Ren, C. R. Abernathy, J. M. Van Hove, P. P. Chow, R. Hickman, J. J. Klaassen, R. F. Kopf, H. Cho, K. B. Jung, J. R. Laroche, R. G. Wilson, J. Han, R. J. Shul, A. G. Baca, and S. J. Pearton, " $300^{\circ} \mathrm{C}$ GaN/AIGaN heterojunction bipolar transistor," MRS Internet J. Nitride Semicond. Res. 3, 41 (1998)

[4] J. Han, A. G. Baca, R. J. Shul, C. G. Willison, L. Zhang, F. Ren, A. P. Zhang, G. T. Dang, S. M. Donovan, X. C. Cao, H. Cho, K. B. Jung, C. R. Abernathy, S. J. Pearton, and R. G. Wilson, “ Growth and fabrication of GaN/AlGaN HBT," Appl. Phys. Lett. 74, 2702 (1999)

[5] O. Ambacher, J. Smart, J. R. Shealy. N. G. Weimann, K. Chu, M. Murphy, W. J. Schaff, L. F. Eastman, R. Dimitrov, L. Wittmer, M. Stutzmann, W. Rieger. and J. Hilsenbeck, “Two-dimensional electron gases induced by spontaneous and piezoelectric polarization charges in $\mathrm{N}$ - and Ga-face AlGaN/GaN heterostructures." J. Appl. Phys 85, 3222 (1999)

[6] P. Kozodoy, M. Hansen, S. P. DenBaars, and U. K. Mishra, "Enhanced Mg doping efficiency in $\mathrm{Al}_{0.2} \mathrm{Ga}_{0.8} \mathrm{~N} / \mathrm{GaN}$ superlattices," ," Appl. Phys. Lett. 74, 3681 (1999)

[7] M.S. Shur, A. D. Bykhovski, and R. Gaska, "2D hole gas induced by piezoelectric and pyroelectric charges," Solid State Electron. 44, 205 (2000); P. M. Asbeck, E. T. Yu. SS. Lau. W. Sun. X. Dang. and C. Shi, "Enhancement of base conductivity via the piezoelectric effect in AlGaN/GaN HBTs," Solid State Electron. 44, 211 (2000)

[8] A. P. Zhang, G. T. Dang, F. Ren, J. Han, A. G. Baca, R. J. Shul, H. Cho, C. Monier, X. A. Cao, C. R. Abernathy, and S. J. Pearton, "DC characteristics of Pnp AlGaN/GaN HBT," Appl. Phys. Lett. (in press) 
[9] E. Alekseev, and D. Pavlidis, "DC and high-frequency performance of AlGaN/GaN HBT," Solid State Electron. 44, 245 (2000)

[10] D. J. H. Lambert, D. E. Lin, R. D. Dupuis, "Simulation of the electrical characteristics of AlGaN/GaN HBT," Solid State Electron. 44, 253 (2000)

[11] Atlas, Silvaco International, Santa Clara, CA (1997)

[12] S. J. Pearton, J. C. Zolper, R. J. Shul , and F. Ren, "GaN: processing, defects, and devices," J. Appl. Phys 86, 1 (1999)

[13] J-S. Jang, I-S. Chang,H-K. Kim, T-Y. Seong, S. Lee, and S-J. Park, "Low-resistance Pt/Ni/Au ohmic contacts to p-type GaN," Appl. Phys. Lett. 74, 70 (1999)

[14] M. Shur, B. Gelmont, and M. Asif Khan, "Electron mobility in two-dimensional electron gas in AlGaN/GaN heterostructres and in bulk GaN," J. Electron. Mat. 25, 777 (1996)

[15] Z. Z. Bandic, P. M. Bridger, E. C. Piquette, and T. C. McGill, "Electron diffusion length and lifetime in p-type GaN," Appl. Phys. Lett. 73, 3276 (1998); Z. Z. Bandic, P. M. Bridger, E. C. Piquette, and T. C. McGill, "Minority carrier diffusion length and lifetime in GaN," Appl. Phys. Lett. 72, 3166 (1998) 


\section{Figure captions}

Figure 1: (a) Calculated collector and base currents as a function of the base-emitter voltage for Npn AlGaN/GaN HBT with different activation energy $\mathrm{E}_{\mathrm{A}}$ values; (b) related DC current gain. The horizontal line represents the case of unity current gain.

Figure 2: Gummel plot and associated DC current gain (inset) of Pnp AlGaN/GaN HBTs in common emitter configuration for several $\mathrm{Mg}$ ionization efficiency conditions.

Figure 3: (a) Collector and base current output simulated from $N p n$ AlGaN/GaN HBTs for several base thickness values as a function of $\mathrm{V}_{\mathrm{BE}}$ voltage; (b) current gain values normalized to the initial $1500 \AA$ structure.

Figure 4: Effect of a quasi-electric field in Npn AlGaN/GaN observed on theoretical (a) Gummel plots and (b) current gain $\beta$.

Figure 5: (a) Influence of the distributed base contact resistance on small-signal performances for conventional $\left(\mathrm{X}_{\mathrm{B}}=1500 \AA \mathrm{A}_{\mathrm{A}}=170 \mathrm{meV}\right) \mathrm{Npn} \mathrm{AlGaN} / \mathrm{GaN}$ HBT. Results from a structure with graded base and optimized contact resistance is displayed; (b) Comparison of unilateral power gain $U$ between a $N p n$ and a $P n p$ AlGaN/GaN HBTs with similar design. 
Fig. 1

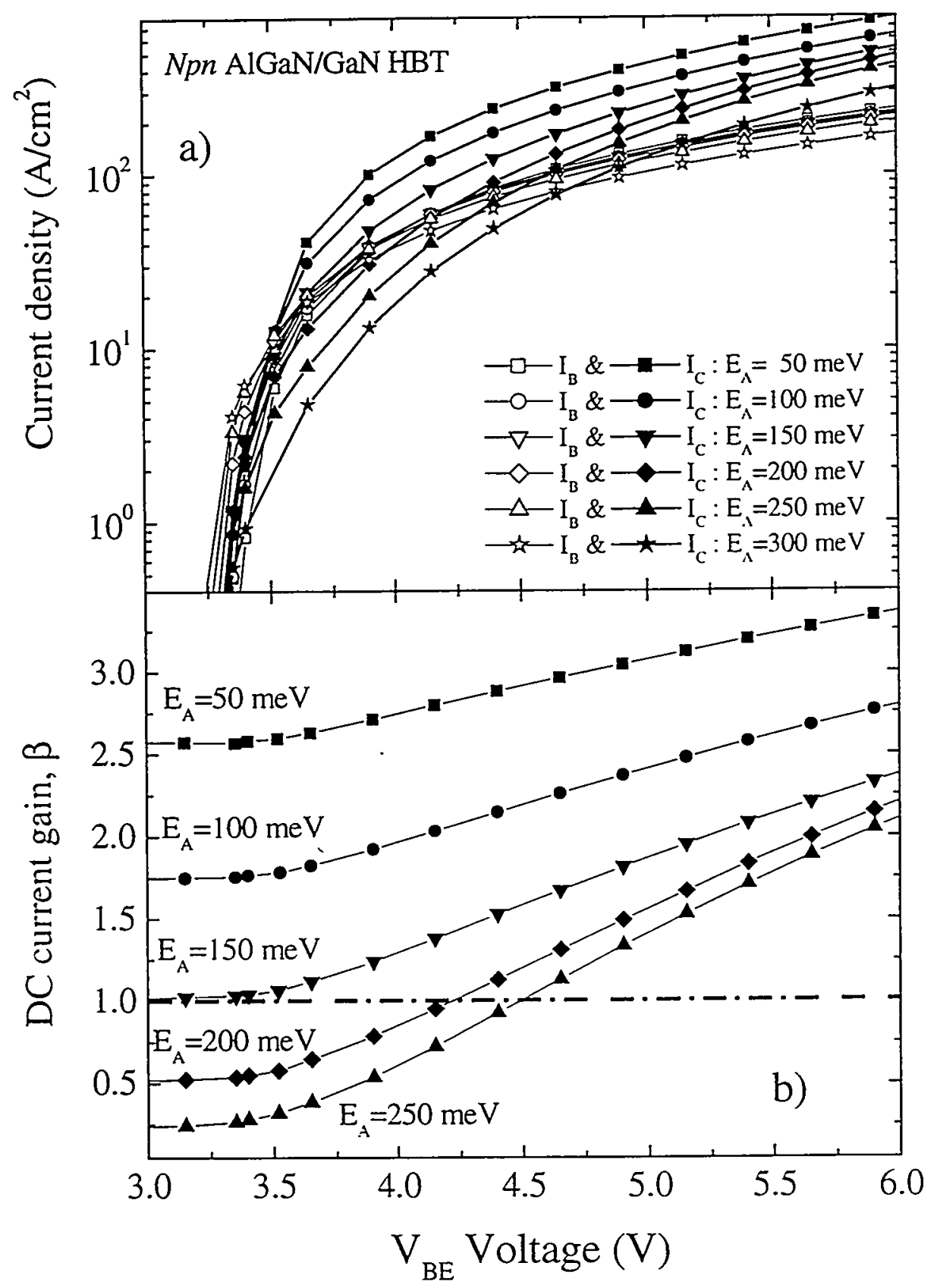


Fig. 2

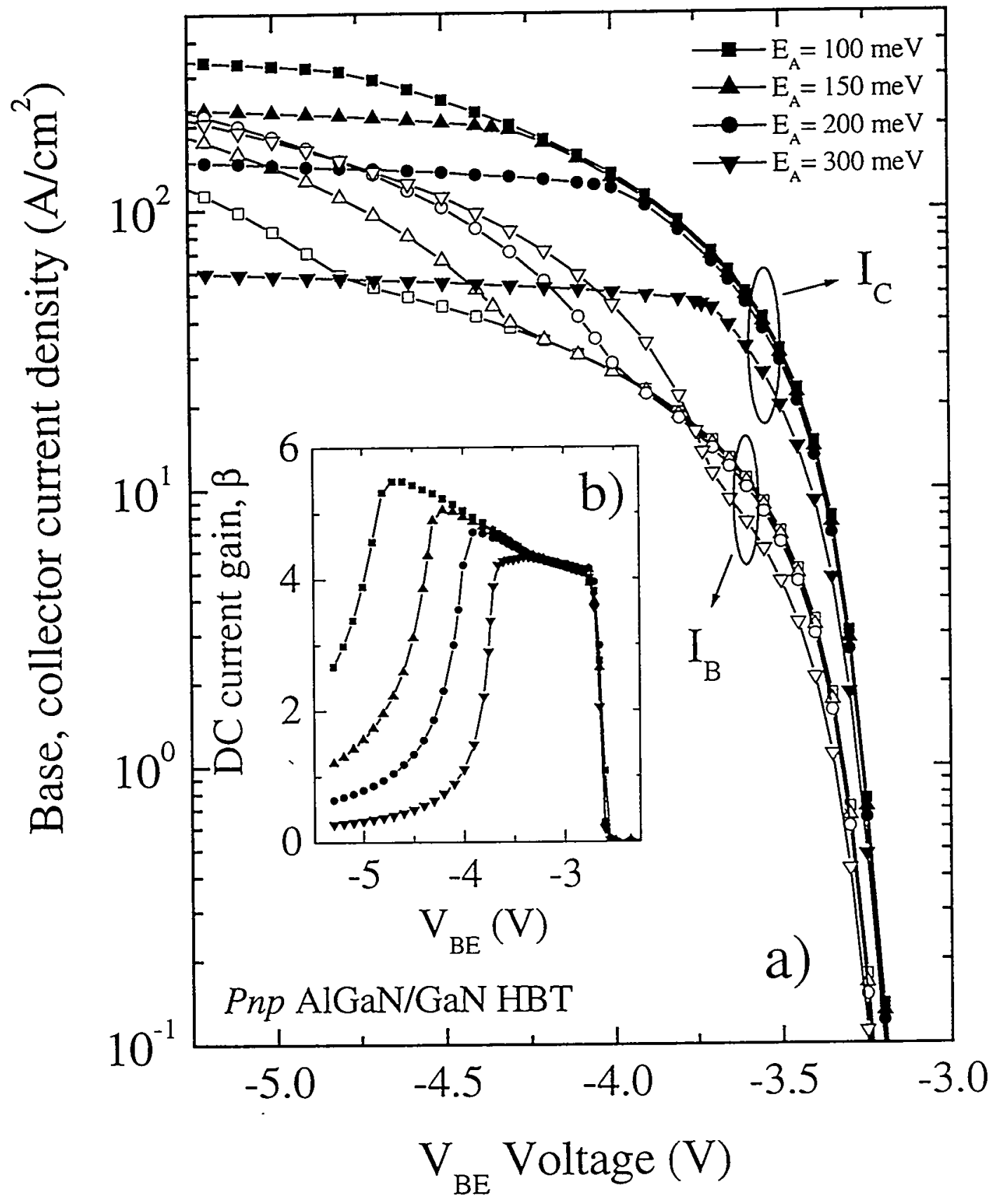


Fig. 3
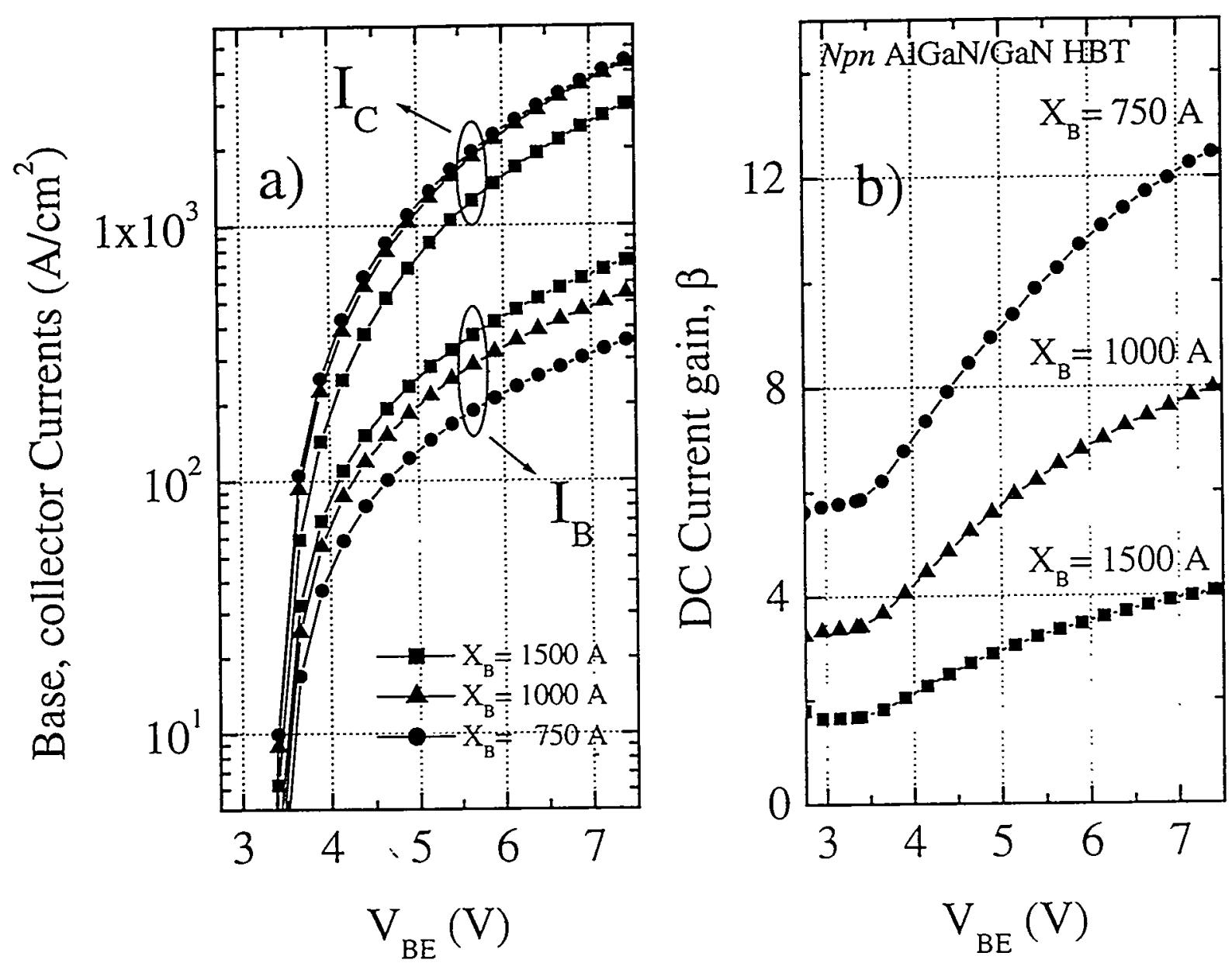
Fig. 4
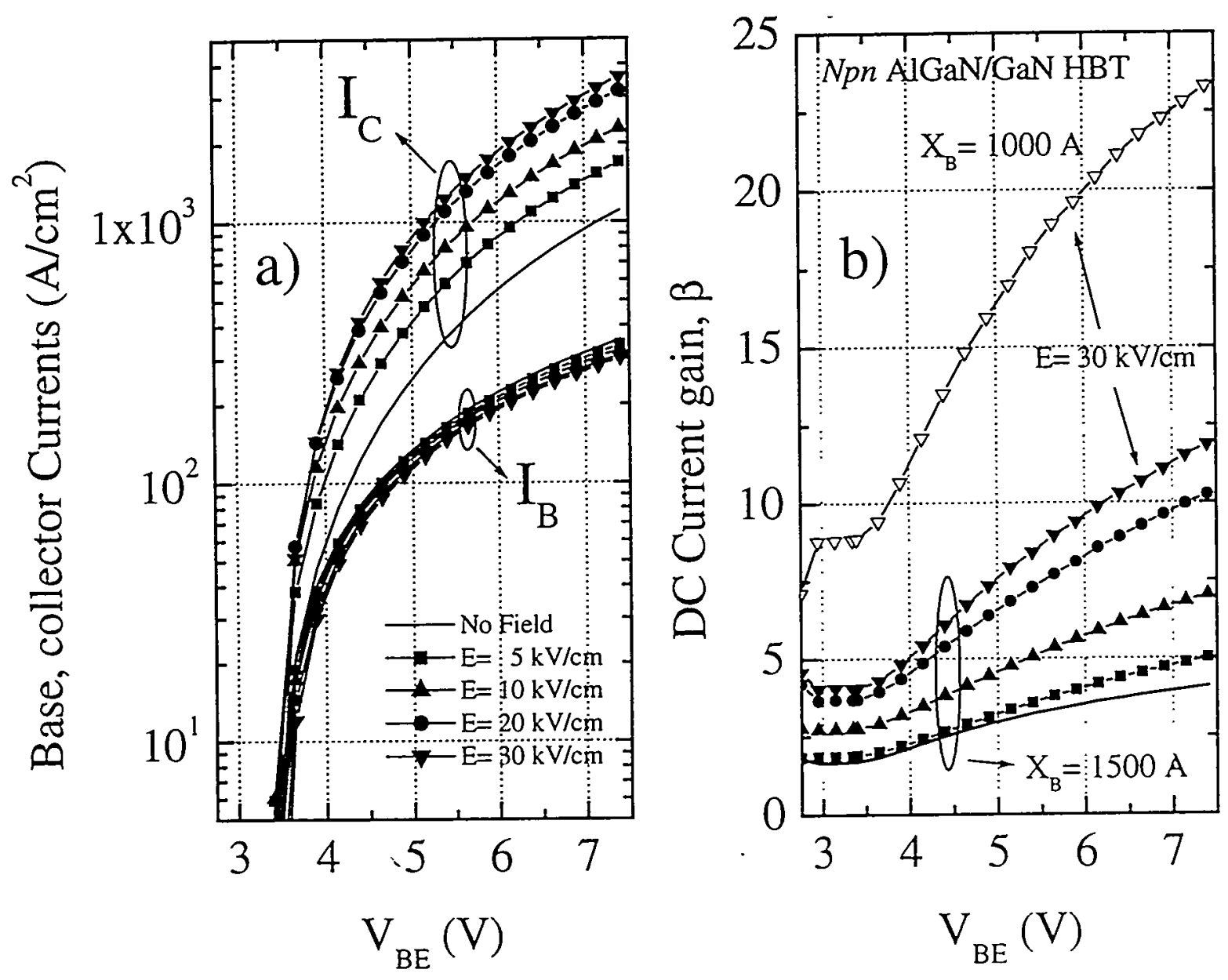
Fig. 5

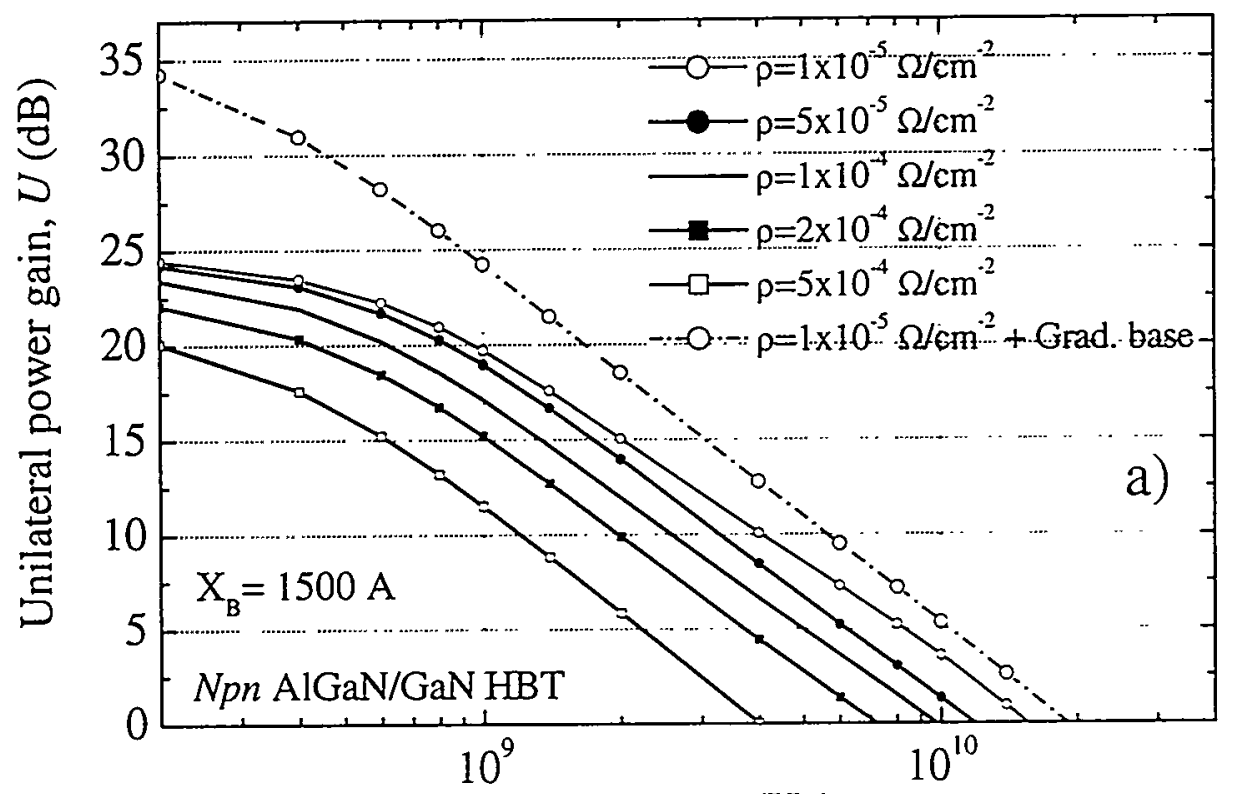

Frequency $(\mathrm{Hz})$

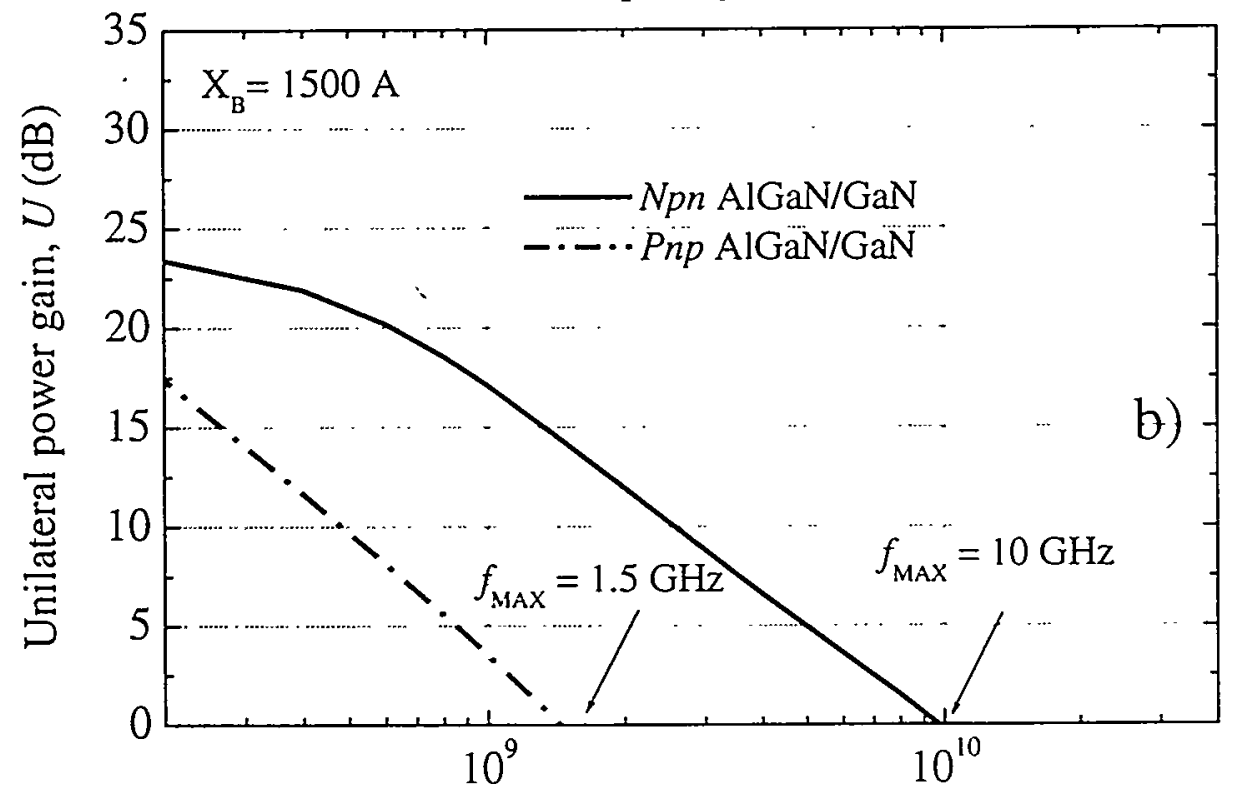

Frequency $(\mathrm{Hz})$ 